\title{
Perfil del Carcinoma Oral de Células Escamosas en el Laboratorio de Anatomía Patológica de la Facultad de Odontología de la Universidad de la República, período 1982-2015
}

\author{
Profile of Oral Squamous Cell Carcinoma in the Pathological \\ Anatomy Laboratory of the Faculty of Dentistry of the \\ University of the Republic, period 1982-2015
}

Perfil do carcinoma epidermoide oral no Laboratório de Anatomia Patológica da Faculdade de Odontologia da

Universidade da República, período 1982-2015

Natalia González Umpiérrez' ORCID: 0000-0001-9006-1680

Verónica Beovide Cortegoso ${ }^{1}$ ORCID: 0000-0003-0888-507X

\section{Resumen}

El Carcinoma oral de células escamosas es la patología oral maligna más frecuente, asociada principalmente al tabaco, alcohol y virus del papiloma humano.

El objetivo del estudio fue determinar el perfil de dicha patología, en el Laboratorio de Anatomía Patológica de la Facultad de Odontología de la Universidad de la República entre 1982-2015. Se realizó un estudio retrospectivo, transversal, analítico. Análisis estadístico usando el modelo de Cox, el test exacto de Fisher y el método de Kaplan Meier. Con 207 casos el 59,9\% fueron hombres y el 98.5\% mayores de 40 años. Localizándose preferentemente en la lengua, con predominio de los carcinomas histopatologicamente bien y moderadamente diferenciados. Se encontró asociación entre el grado histopatológico, género y edad; con un tiempo medio de sobrevida de 2.36 ańos y una tasa de sobrevida del $43 \%$ a los 5 años. El estudio permitió conocer dicha patología en un servicio de referencia y generar una base de datos para posteriores estudios.

Palabras clave: Carcinoma de células escamosas de cabeza y cuello, Epidemiología y Sobrevida.

Cátedra de Anatomía Patológica de la Facultad de Odontología de la Universidad de la República, Montevideo, Uruguay. 


\section{Abstract}

Oral squamous cell carcinoma is the most frequent malignant oral pathology, mainly associated with tobacco, alcohol and human papilloma virus.

The objective of the study was to determine the profile of this pathology, in the Laboratory of Pathological Anatomy of the Faculty of Dentistry of the University of the Republic between 1982-2015. A retrospective, cross-sectional, analytical study was carried out. Statistical analysis using the Cox model, Fisher's exact test and the Kaplan Meier method. With 207 cases $59.9 \%$ were men and $98.5 \%$ older than 40 years. Preferably located on the tongue, with a predominance of histopathologically well and moderately differentiated carcinomas. An association was found between histopathological grade, gender, and age; with an average survival time of 2.36 years and a survival rate of $43 \%$ at 5 years. The study allowed knowing this pathology in a reference service and generating a database for subsequent studies.

Keywords: Head and neck squamous cell carcinoma, Epidemiology and Survival.

\section{Introducción y antecedentes}

El carcinoma oral de células escamosas (COCE) es la patología oral maligna de mayor frecuencia, representa del 2 al 3\% de todas las neoplasias malignas y hasta el $80-90 \%$ de las que asientan en la cavidad bucal ${ }^{(1-8)}$.

El COCE es un tumor sólido maligno, que se origina en el epitelio escamoso estratificado de la mucosa del tracto aerodigestivo superior, se caracteriza por presentar diferentes grados de diferenciación histológica y un elevado potencial invasivo y metastásico ${ }^{(9)}$; su etiología se

\section{Resumo}

O carcinoma epidermóide de boca é a patologia maligna mais frequente, principalmente associada ao tabaco, álcool e papiloma vírus humano.

O objetivo do estudo foi determinar o perfil dessa patologia, no Laboratório de Anatomia Patológica da Faculdade de Odontologia da Universidade da República entre 1982-2015. Foi realizado um estudo analítico retrospectivo, transversal. Análise estatística pelo modelo de Cox, teste exato de Fisher e método de Kaplan Meier. Em 207 casos, 59,9\% eram homens e 98,5\% tinham mais de 40 anos. De preferência localizado na língua, com predominância de carcinomas histopatologicamente bem e moderadamente diferenciados. Foi encontrada associação entre grau histopatológico, sexo e idade; com tempo médio de sobrevivência de 2,36 anos e taxa de sobrevivência de 43\% aos 5 anos. $\mathrm{O}$ estudo permitiu conhecer essa patologia em um serviço de referência e gerar um banco de dados para estudos subsequentes.

Palavras-chave: Carcinoma espinocelular de cabeça e pescoço, Epidemiologia e Sobrevivência.

asocia principalmente a factores de riesgo como el tabaco, alcohol y virus de papiloma humano 16 y 18 (VPH) ${ }^{(10-13)}$.

En 2018 el Global Cancer Observatory (GLOBOCAN) reporta los siguientes datos para Uruguay: en una población de 3.469 .551 se registraron 15.101 nuevos casos de cáncer, con una mortalidad de 8.589 y una prevalencia en 5 años de 35.478; según fuentes del Registro Nacional de Cáncer (RNC) y la Organización Mundial de la Salud (OMS) ${ }^{(14)}$.

La situación epidemiológica del Uruguay en relación al cáncer, publicada recientemente por el 
RNC reporta los siguientes datos a nivel bucofaríngeo para el período 2011-2015 (expresado en tasas ajustadas cada 100.000 habitantes): un total de 1.529 nuevos casos (1.107 hombres y 422 mujeres), localizado con mayor frecuencia en la lengua (378 casos) sin distinción de sitio anatómico lingual, una incidencia de 10.17 y 2.81 para hombres y mujeres respectivamente y mortalidad 6.67 en hombres y 1.21 en mujeres (15).

Morfológicamente el COCE se presenta como una proliferación neoplásica epitelial maligna, conformada por nidos, cordones e islotes que recuerdan en mayor o menor grado al epitelio escamoso de la mucosa de revestimiento de la cual derivan. Actualmente la tipificación histológica del COCE se realiza en base a la clasificación histológica de la OMS y al frente de invasión tumoral; en COCE bien diferenciados, moderadamente diferenciados e indiferenciados ${ }^{(7,8)}$.

El COCE es una neoplasia agresiva con un comportamiento biológico impredecible y un pronóstico desfavorable ${ }^{(16)}$. La agresividad de esta neoplasia maligna se evidencia en que a pesar de las estrategias terapéuticas disponibles en la actualidad, que incluyen su extirpación quirúrgica ya sea en forma exclusiva o en combinación con radioterapia y/o quimioterapia, la tasa de sobrevida y la tasa de recurrencia a los 5 años es deficiente, sobreviviendo solo el 50\% de los pacientes diagnosticados y tratados ${ }^{(17-19)}$, situación que se ha mantenido inmodificable en las últimas cinco décadas ${ }^{(20-22)}$. El pronóstico se relaciona a factores como el tamaño tumoral, la presencia de ganglios linfáticos metastásicos, el grado de diferenciación tumoral, la invasión de estructuras adyacentes, la localización y el tratamiento realizado ${ }^{(23,24)}$. El sistema de estadificación TNM del inglés TNM Staging System, donde $\mathrm{T}$ significa tumor primario, $\mathrm{N}$ ganglios linfáticos regionales y $\mathrm{M}$ presencia de metástasis a distancia, es un método universal de estadificación de la enfermedad en el paciente con cáncer, el cual permite la toma de decisiones terapéuticas. Fue actualizado en 2017 para cada topografía, además de identificarse los COCE $\mathrm{VPH}+\mathrm{yPH}^{(25)}$.

El objetivo de este estudio fue determinar el perfil del COCE en el Laboratorio de Anatomía Patológica de la Facultad de Odontología (LAPFO) de la Universidad de la República (UdelaR), en el periodo 1982-2015. Describiendo la distribución de acuerdo a los parámetros clínico-morfológicos (edad, género, localización y grado histopatológico) y determinar la tasa de sobrevida.

\section{Metodología}

\section{1- Consideraciones éticas:}

El proyecto cuenta con la aprobación del comité de ética de la Facultad de Odontología - UdelaR Montevideo -Uruguay. (Expediente 163/16).

\section{2- Diseño del estudio:}

Estudio retrospectivo, transversal y analítico, tipo de muestreo no probabilístico por conveniencia de acuerdo a los criterios de inclusión y exclusión propuestos para el trabajo. La población comprendió todos los registros biópsicos del LAPFO (11.321) durante el periodo 1982-2015 y la muestra se constituyó por todos los casos con diagnóstico histopatológico de COCE de la mucosa bucal. Del total de registros biópsicos en dicho período (11.321) se obtuvo una muestra de 207 casos de COCE $(1,8 \%)$ que cumplieron con los criterios de inclusión y exclusión planteados a continuación.

\section{3- Criterios de inclusión y exclusión:}

Criterios de inclusión: COCE con datos clínico morfológicos completos, donde se registren las variables: edad, género, localización y grado histopatológico de la lesión. Se aceptó como diagnóstico histopatológico de COCE el registrado en el informe biópsico que cumplieran con los criterios histológicos de malignidad planteados por la OMS (bien diferenciados, 
moderadamente diferenciados e indiferenciados), se consideró la posibilidad de una reevaluación del diagnóstico histopatológico en aquellos casos que el grado histopatológico no estuviera detallado según los criterios establecidos (no fue necesaria la reevaluación en ningún caso); en los casos que para un paciente existieran dos registros (biopsia incisional y excisional), fue contabilizado como un solo caso (para evitar la duplicación de casos) y la graduación histológica registrada del COCE fue la detallada en la biopsia excisional, mientras que para la fecha de inicio de la enfermedad se consideró la registrada en la primer biopsia con diagnóstico de COCE.

Para determinar la tasa de sobrevida de los pacientes con COCE se constituyó una muestra, donde el criterio de inclusión fue que el registro contara con la cédula de identidad del paciente, dato necesario para ser localizados en la base de datos del RNC, $(\mathrm{n}=99)$.

Criterios de exclusión: Se excluyeron los casos con datos incompletos, la ausencia de alguna de las variables (edad, género, localización y graduación histológica).

\section{4- Análisis estadístico:}

Se realizó estadística descriptiva, se buscó establecer relaciones significativas entre los diferentes parámetros clínico-morfológicos mediante el test exacto de Fisher. Se construyeron curvas de sobrevida mediante la metodología de Kaplan Meier. Las pruebas de hipótesis fueron realizadas a un nivel de 5\% y los cálculos en el software de uso libre $\mathrm{R}$ utilizando la librería survival. Atendiendo al posible efecto del género, edad, grado histopatológico y localización agrupada de los tumores se realizó el análisis mediante el modelo de riesgos proporcionales de Cox. La selección de las variables fue llevada a cabo mediante un procedimiento back Ward, luego de ajustados los modelos se verificó la validez del supuesto de proporcionalidad.

\section{Resultados}

Los resultados se muestran en el Cuadro 1, se registró un rango etario entre 16 y 99 ańos, con una media de 63 ańos $\pm 12.57,41.5 \%$ (86) entre los 41-60 años y 47.8\% (99) entre los 61-80 años. El 59.9\% (124) fueron masculinos, con una relación m: f de 1.5: 1. En relación al grado histopatológico, 44.4\% (92) fueron carcinomas bien diferenciados, $44.9 \%$ (93) moderadamente diferenciados y $10.6 \%$ (22) indiferenciados. La localización más frecuente fue la lengua con $26.6 \%$ (55) y el reborde alveolar con un 23.7\% (49).

\section{Cuadro 1. Frecuencias univariadas del COCE} $(\mathbf{n}=207)$

\begin{tabular}{|c|c|c|}
\hline & $\begin{array}{l}\text { Frecuencia } \\
\text { absolutas }\end{array}$ & $\begin{array}{c}\text { Frecuencias } \\
\text { relativas }\end{array}$ \\
\hline $\begin{array}{l}\text { Grupo etario } \\
\text { (media } 63,63 \pm 12,57 \text { ) }\end{array}$ & $\mathbf{n}$ & $\%$ \\
\hline menos de 20 años & 1 & 0.5 \\
\hline de 21 a 40 años & 2 & 1.0 \\
\hline de 41 a 60 años & 86 & 41.5 \\
\hline de 61 a 80 años & 99 & 47.8 \\
\hline 810 más años & 19 & 9.2 \\
\hline Género & $\mathbf{n}$ & $\%$ \\
\hline Femenino & 83 & 40.1 \\
\hline Masculino & 124 & 59.9 \\
\hline Grado Histopatológico & $\mathbf{n}$ & $\%$ \\
\hline Bien diferenciados & 92 & 44.4 \\
\hline Moderadamente diferenciados & 93 & 44.9 \\
\hline Indiferenciados & 22 & 10.6 \\
\hline Localización agrupada & $\mathbf{n}$ & $\%$ \\
\hline Encía & 6 & 2.9 \\
\hline Mucosa labial & 6 & 2.9 \\
\hline Lengua & 55 & 26.6 \\
\hline Mucosa yugal & 26 & 12.6 \\
\hline Paladar & 31 & 15.0 \\
\hline Piso de boca & 22 & 10.6 \\
\hline Reborde alveolar & 49 & 23.7 \\
\hline Trígono retromolar & 12 & 5.8 \\
\hline Total & 207 & 100 \\
\hline
\end{tabular}


Al realizar la asociación entre variables se observó que el grado histopatológico en relación al género y la edad fueron significativos, mostrando que los hombres tienen mayor proporción de COCE moderadamente diferenciado y las mujeres mayor proporción de COCE bien diferenciado (p-valor de 0.031) (Tabla 1); en cuanto a la edad, los mayores de 81 años tienen mayor proporción de COCE indiferenciado (pvalor 0.027) (Tabla 2).

El análisis multivariado en relación a la localización no mostró asociación significativa, posiblemente debido a las múltiples localizaciones del COCE en la mucosa bucal, en este estudio se agruparon en 8 categorías. En las mujeres las localizaciones de mayor frecuencia fueron el reborde alveolar 28.9\% (24) y la lengua 26.5\% (22), mientras que en los hombres la lengua fue $26.6 \%$ (33) y $20.2 \%$ (25) el reborde alveolar.

Para el análisis de sobrevida se trabajó con la muestra de 99 pacientes, entre los cuales se observó el evento de fallecimiento en 57 casos, el resto correspondieron a censuras. Se construyó la curva de sobrevida (Fig. 1), a partir de la cual se estimó que el tiempo medio de sobrevida fue de 2.36 años con un intervalo de confianza
$(1.60-8.24)$ y la tasa de sobrevida a los 5 años fue del $43 \%$, sin considerar los estadios del sistema TNM debido al no registro de los mismos en los archivos.

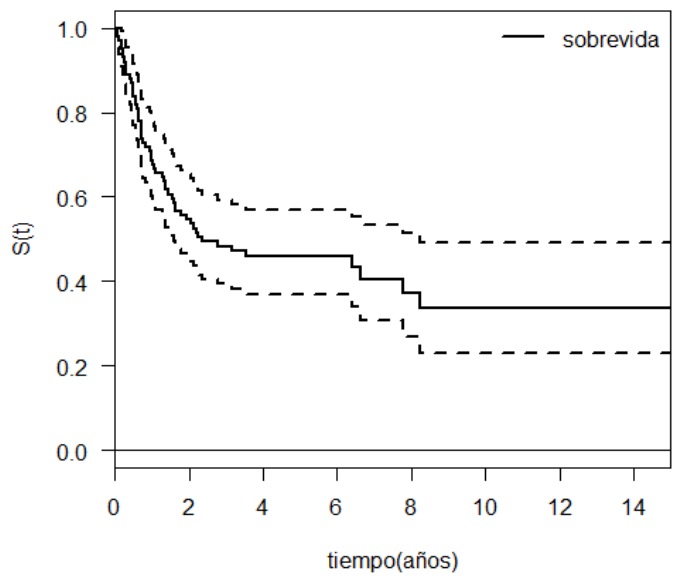

Fig.1. Curva de sobrevida, Kaplan-Meier.

$\mathrm{Al}$ asociar el riesgo de fallecimiento en función del género, edad, grado histopatológico y localización, se encontró asociación estadísticamente significativa con el grado histológico. El modelo final se presenta en el Cuadro 2, donde se observa que los pacientes con diagnóstico de

Tabla 1. Asociación entre grado histopatológico y género

\begin{tabular}{|c|c|c|c|c|}
\hline & Bien diferenciado $n(\%)$ & $\begin{array}{c}\text { Moderadamente diferenciado } \\
\mathrm{n}(\%)\end{array}$ & $\begin{array}{c}\text { Indiferenciado } \\
\mathrm{n}(\%)\end{array}$ & Total $\mathrm{n}(\%)$ \\
\hline Femenino & $46(55.4)$ & $27(32.5)$ & $10(12)$ & $83(100)$ \\
\hline Masculino & $46(37.1)$ & $66(53.2)$ & $12(9.7)$ & $124(100)$ \\
\hline Total & $92(44.4)$ & $93(44.9)$ & $22(10.6)$ & $207(100)$ \\
\hline
\end{tabular}

Test exacto de Fisher, p-valor: 0,031

Tabla 2. Asociación entre grado histopatológico y edad

\begin{tabular}{|c|c|c|c|c|}
\hline & $\begin{array}{c}\text { Bien diferenciado } \\
\mathrm{n}(\%)\end{array}$ & $\begin{array}{c}\text { Moderadamente diferenciado } \\
\mathrm{n}(\%)\end{array}$ & $\begin{array}{c}\text { Indiferenciado } \\
\mathrm{n}(\%)\end{array}$ & $\begin{array}{c}\text { Total } \\
\mathrm{n}(\%)\end{array}$ \\
\hline Edad menos de 60 años & $40(44.9)$ & $43(48.3)$ & $6(6.7)$ & $89(100)$ \\
\hline de 61 a 80 años & $44(44.4)$ & $43(43.4)$ & $12(12.1)$ & $99(100)$ \\
\hline 81 años o más & $8(42.1)$ & $7(36.8)$ & $4(21.1)$ & $19(100)$ \\
\hline Total & $92(44.4)$ & $93(44.9)$ & $22(10.6)$ & $207(100)$ \\
\hline
\end{tabular}

Test exacto de Fisher, p-valor: 0,027 
COCE moderadamente diferenciados presentan casi el doble de riesgo de fallecer que los pacientes con COCE bien diferenciado ( $p$-valor 0,031). Al analizar los COCE indiferenciados se observó el evento de fallecimiento en el total de los casos, a los 7 años aproximadamente del seguimiento, a diferencia de los COCE bien diferenciados que al final del estudio contaban aún con pacientes vivos (Fig. 2).

Cuadro 2. Modelo final de riesgo proporcional de COX

\begin{tabular}{|c|c|c|c|}
\hline & Coeficiente & Riesgo & p-valor \\
\hline COCE Moderadamente diferenciado & 0,679 & 1,971 & 0,031 \\
\hline COCE indiferenciado & 0,086 & 0,109 & 0,860 \\
\hline
\end{tabular}

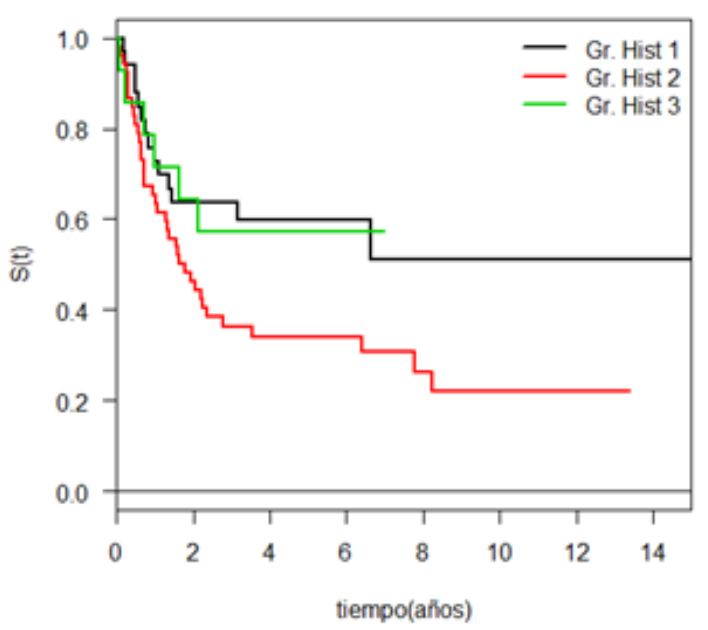

Fig, 2. Curvas de sobrevida según grado histopatológico

\section{Discusión}

El estudio del COCE es de gran importancia debido a su alta morbilidad y mortalidad, siendo de gran utilidad obtener información relevante que permita comparar resultados con otros centros especializados en patología bucal, aportando conocimiento sobre la sobrevida y las características clínico-patológicas.

El presente estudio permitió obtener información institucional para la FO de la UdelaR, es- tableciendo el perfil del COCE en el LAPFO, obteniendo una base de datos importante para posteriores estudios.

En nuestro trabajo tuvimos algunas limitantes debido a la falta de registro adecuado en las fichas anátomo-patológicas, lo que dificulta la estadificación TNM y la evaluación de factores de riesgo. Aun así destacamos la importancia de contar con una cohorte de 99 pacientes para el análisis de sobrevida.

En este estudio, el 59.9\% de los COCE afectó a los hombres, dato que coincide con la literatura (19,21,26,27); sin embargo se encontró diferencias en cuanto a la relación m: f 1.5: 1 de nuestro estudio con los mostrados por el Instituto $\mathrm{Na}$ cional de Cáncer (INCA) en Brasil que reporto una proporción mayor de 3.7: $1^{(28)}$ y los recientemente aportados por el RNC en Uruguay que publicó una proporción 3.6: 1 para hombres y mujeres respectivamente ${ }^{(15)}$. La mayor frecuencia en hombres coincide con lo reportado en GLOBOCAN $2018^{(14)}$ y otros autores, donde se evidencia que el COCE es dos o tres veces más frecuente en hombres en la mayoría de los grupos étnicos, con cierta tendencia en los últimos años al aumento del COCE en las mujeres debido al mayor consumo de tabaco, dicho dato podría explicar la relación 1,5: 1 obtenida en nuestro estudio ${ }^{(2,19,29)}$.

Un trabajo retrospectivo publicado en 2014 por Momares y col. con 217 pacientes diagnosticados con COCE entre 1989 y 2010, del departamento de Patología Oral de la Escuela de Odontología Universidad Mayor de Chile, mostró resultados similares a los obtenidos en nuestro estudio, al compararlos se encontró que la distribución por género, la proporción entre ambos, el rango etario y el promedio de edad fueron casi idénticos. La lengua y el reborde alveolar fueron las localizaciones más frecuentes para ambos, con algunas diferencias en el resto de las localizaciones. Respecto al grado de diferenciación histológico ambos estudios encontraron que los COCE bien diferenciados y 
moderadamente diferenciados fueron más prevalentes que los COCE indiferenciados ${ }^{(30)}$.

$\mathrm{Al}$ analizar la edad de presentación del COCE, en nuestro trabajo la media fue de $63 \pm 12,57$ años con un rango etario de 16 a 99 años, una frecuencia de 41,5\% de los COCE entre los 41 y 60 ańos, aumentando a 47,8 \% en el grupo de 61-80 ańos. Oliveira y col. en 2015 reportan en un Hospital Público de Uruguay un promedio de $60,75 \pm 11,26$ años, similar al observado ${ }^{(19)}$. La literatura reporta que más del $90 \%$ se diagnostican en mayores de 40 años, y más del 50\% en individuos con más de 65 ańos, en nuestro estudio el $98.5 \%$ se presentó en individuos mayores de 40 años y el $57 \%$ en mayores de 60 años ${ }^{(31-33)}$.

Si analizamos la edad de presentación del COCE según el género en nuestro estudio, podemos destacar que el $80 \%$ de las mujeres y el $96 \%$ de los hombres tienen entre 41 y 80 años, de esos grupos más del $50 \%$ se encuentran en el rango de mayor edad (61 a 80 años). En el análisis de los rangos etarios observamos que en el grupo de menores de 40 años no existían registros de COCE en hombres, mientras que en el grupo de mayores de 81 años, las mujeres registran un número casi tres veces mayor a los hombres.

La localización de preferencia fue la lengua con un $26.6 \%$, en concordancia con un trabajo realizado en el Laboratorio de Anatomía Patológica del INCA del Uruguay en 2017, como lo reportado en la literatura en la mayoría de los trabajos sobre COCE ${ }^{(34)}$. A diferencia con lo visto en la segunda localización más frecuente (reborde alveolar con 23,7\%), donde la mayoría de los estudios reporta como segunda localización al piso de boca y/o paladar, esta diferencia puede estar vinculada a la forma de agrupar las localizaciones del COCE ${ }^{(35-37)}$.

Al analizar el grado de diferenciación histopatológico encontramos resultados similares a los obtenidos en nuestro estudio, Wei-ping Jie y col. reportan el $90,8 \%$ de los casos fueron los COCE bien diferenciados y moderadamen- te diferenciados ${ }^{(38)}$. Otros estudios mostraron datos similares, Sairay col. en Pakistán para el período 2015-2016 mostró que el 52\% de los COCE fueron moderadamente diferenciados y el $42 \%$ bien diferenciados ${ }^{(39)}$, mientras Gul y col. obtuvieron en un Hospital terciario del sur de Punjab India, un $43 \%$ de pacientes con COCE bien diferenciado, 38\% moderadamente diferenciado y $20 \%$ con COCE indiferenciado ${ }^{(40)}$. Leite y col. en 2018 en un estudio de Brasil de COCE en una población de bajo desarrollo económico, $\mathrm{n}=194$ casos, el 54,6\% fueron bien diferenciados, $37,1 \%$ moderadamente diferenciados y 5,2\% carcinomas indiferenciados, este estudio reporta un perfil similar a nuestro estudio ${ }^{(41)}$.

Al realizar la asociación entre las variables, se observa significancia estadística del grado histopatológico del COCE en relación al género y edad. Los hombres presentaron mayor proporción de carcinomas moderadamente diferenciados y las mujeres mayor proporción de COCE bien diferenciados; indicando que a mayor edad, mayor proporción de carcinomas indiferenciados, similar a lo encontrado en un estudio publicado en 2010 sobre las Características clínico-histopatológicas del COCE en el Hospital Universitario San Vicente de Paúl, Colombia ${ }^{(42)}$.

La sobrevida de los pacientes con COCE a los 5 años fue de $43 \%$, similar a lo reportado por Momares y col. en Chile que fue del $46 \%$, mientras que el tiempo medio de sobrevida para ellos fue de 6,9 años, superior al encontrado en nuestro estudio que fue de 2.36 años ${ }^{(30)}$. Otros autores reportan para el COCE una sobrevida que va del $30 \%$ a $50 \%{ }^{(33,43)}$.

Oliveira y col. muestran una tasa de sobrevida global aún menor, con una supervivencia a los 5 años del 24\% ${ }^{(19)}$. Bonfante y col. en 2014 en un estudio nacional del Sistema Único de Salud de Brasil en el período 2000-2006, reportan datos de sobrevida específica a los 5 años para el cáncer de boca de 4,6 años y una sobrevida global a los 5 años de 2,6 años similar a nuestro 
estudio (2,36 años) ${ }^{(44)}$. Otra investigación con 274 casos de la Universidad de Buenos Aires (Argentina) que evaluó la sobrevida global para el cáncer de boca, reportó una tasa del 39\% a los 5 años ${ }^{(45)}$.

\section{Conclusiones}

En general el estudio del COCE en Uruguay es escaso, principalmente en lo referido al pronóstico y sobrevida de los pacientes, estudios retrospectivos como el realizado cuentan con limitaciones importantes debido a la falta de registros completos, ausencia en la mayoría de los casos de datos clínicos relevantes para establecer un sistema de estatificación TNM y fundamentalmente a la falta de seguimiento de los pacientes. De todas maneras estos estudios descriptivos sirven como fuente de hipótesis para posteriores estudios analíticos.

Considerando la alta mortalidad y morbilidad del COCE, los profesionales de la salud deberíamos considerar la importancia de la detección temprana de estas lesiones y la educación sobre los factores de riesgo ya conocidos, como el tabaco, alcohol e infección con el VPH, teniendo en cuenta que uno de los factores más importantes en relación al mal pronóstico del COCE es el diagnóstico tardío.

\section{Contribución de autoría}

1. Concepción y diseño del estudio

2. Adquisición de datos

3. Análisis de datos

4. Discusión de los resultados

5. Redacción del manuscrito

6. Aprobación de la versión final del manuscrito

NGU ha contribuido en $1,2,3,4,5$ y 6 . VBC ha contribuido en $1,4,5$ y 6 .

\section{Referencias}

1. Maraboli-Contreras S, Adorno-Farias D, Maturana-Ramirez A, Rojas-Alcayaga G, FuentesAlburquenque M, Espinoza-SantanderI. Sobrevida de carcinoma oral de células escamosas: reporte de la Universidad de Chile.Rev. Clin. Periodoncia Implantol. Rehabil. Oral. 2018; 11 (3): $147-151$.

2. Torre LA, Bray F, Siegel RL, Ferlay J, LortetTieulent J, Jemal A. Global cancer statistics, 2012. CA Cancer J Clin. 2015; 65 (2): 87-108.

3. Warnakulasuriya S. Global epidemiology of oral and oropharyngeal cancer. Oral Oncology. 2009; 45: 309-316.

4. Neville, Brad W. Patologia oral e maxilofacial. 4. ed. Rio de Janeiro: Elsevier, 2016. 912 p. ISBN: 978-85-352-6564-4

5. Licitra L, Locati LD, Bossi P, Cantù G. Head and neck tumors other than squamous cell carcinoma. Curr Opin Oncol. 2004; 16 (3): 23641.

6. Johnson NW, Jayasekara P, Amarasinghe AAHK. Squamous cell carcinoma and precursor lesions of the oral cavity: epidemiology and etiology. Periodontol 2000. 2011 ; 57 (1): 1937.

7. Barnes L, Eveson JW, Reichart P, Sidransky D. World Health Organization classification of tumours: Pathology \& genetics Head and Neck tumours. International Agency for Research on Cancer, Lyon, France 2005; 168-175.

8. El-Naggar AK, Chan JKC, Rubin Grandis J, Takata T, Slootweg PJ. WHO classification of head and neck tumours. International Agency for Research on Cancer. 4th ed. 2017.

9. Kang H, Kiess A, Chung C. Emerging biomarkers in head and neck cancer in the era of genomics. Nat Rev Clin Oncol. 2015; 12: 11-26.

10. Miguel Cruz PA, Niño Peña A, Batista Marrero K, Miguel-Soca PE. Factores de riesgo de cáncer bucal. Rev Cubana Estomatol. 2016; 53 (3): 128-45.

11. Jiang X, Wu J, Jiexue Wang RH. Tobacco and oral squamous cell carcinoma: A review of carcinogenic pathways. Tob Induc Dis. 2019; 17.

12. Auluck A, Hislop G, Bajdik C, Poh C, Zhang $\mathrm{L}$ RM. Trends in oro- pharyngeal and oral cavity cancer incidence of human papillomavirus (HPV)-related and HPV-unrelated sites in a 
multicultural population: the British Columbia experience. Cancer. 2010; 116: 2635-2644.

13. Young D, Xiao CC, Murphy B, Moore M, Fakhry C, Day TA. Increase in head and neck cancer in younger patients due to human papillomavirus (HPV). Oral Oncology. 2015; 51.

14. Ferlay J, Colombet M, Soerjomataram I, Mathers C, Parkin DM, Piñeros M, Znaor A, Bray F. Estimating the global cancer incidence and mortality in 2018: GLOBOCAN sources and methods. Int J Cancer. 2018. https: //doi. org/10.1002/ijc.31937

15. Comisión Honoraria de Lucha Contra el Cáncer. Situacion Epidemiologica del Uruguay en relacion al Cancer. Regist Nac del Cáncer, Com Honor Lucha Contra el Cáncer. 2019; 1-61.

16. Lopes KM,Souza JA, Lacerda de Souza L, Akiko ML, Guimarão DM, Pontes HA. La proteína Ki-67 predice la supervivencia en las células de carcinoma escamoso oral: un estudio inmunohistoquímico. Braz. res oral. 2017; 31: e66.

17. Majumdar B, Shankargouda P, Sachin CS, Gargi SS and Roopa SR. Clinico-pathological prognosticators in oral squamous cell carcinoma: An update Translational. Research in Oral Oncology. 2017; (2): 1-14.

18. Mamelle G, Pampurik J, Luboinski B, Lancar R, Lusinchi A, Bosq J. Lymph node prognostic factors in head and neck squamous cell carcinomas. Am J Surg. 1994; 168 (5): 494-8.

19. Oliveira MLC, Wagner VP, Sant'ana Filho M, Carrard VC, Hugo FN, Martins MD. A 10year analysis of the oral squamous cell carcinoma profile in patients from public health centers in Uruguay. Braz Oral Res. 2015; 29 (1): 1-8.

20. Barrios E, Garau M. Cáncer: magnitud del problema en el mundo y en Uruguay, aspectos epidemiológicos. An la Fac Med. 2017; 4 (1): 04-66.

21. Barrios E, Garau M, Alonso R, Musetti C. IV atlas de incidencia del cancer en el Uruguay. Registro Nacional de Cáncer, Comisión Honoraria de lucha contra el Cáncer, 2014.

22. Ferlay J, Soerjomataram I, Dikshit R, Eser S, Mathers C, Rebelo M, et al. Cancer incidence and mortality worldwide: Sources, methods and major patterns in GLOBOCAN 2012. Int J Cancer. 2015; 136 (5).

23. Patel RS, Clark JR, Dirven R, Wyten R, Gao $\mathrm{K}$, O’Brien CJ. Prognostic factors in the surgi- cal treatment of patients with oral carcinoma. ANZ J Surg. 2009 Jan; 79(1-2): 19-22.

24. Mamelle G, Pampurik J, Luboinski B, Lancar R, Lusinchi A, Bosq J. Lymph node prognostic factors in head and neck squamous cell carcinomas. Am J Surg. 1994; 168(5): 494-8.

25. Lydiatt WM, Patel SG, O'Sullivan B, Brandwein MS, Ridge JA, Migliacci JC, et al. Head and neck cancers-major changes in the American Joint Committee on cancer eighth edition cancer staging manual. CA Cancer J Clin. 2017; 67(2): 122-37.

26. Roberto De Oliveira L, Ribeiro-Silva A, Zucoloto S. Perfil da incidência e da sobrevida de pacientes com carcinoma epidermóide oral em uma população brasileira Incidence and survival profile of patients with oral squamous cell carcinoma in a Brazilian population. J Bras Patol Med Lab. 2006; 42 (5): 385-92.

27. Ferlay J, Colombet M, Soerjomataram I, Mathers C, Parkin DM, Piñeros M, A Znaor $\mathrm{F}$ Bray. Estimating the global cancer incidence and mortality in 2018: GLOBOCAN sources and methods. Int J Cancer. 2018; 144 (8): 1941 1953

28. Estimativa 2018: incidência de câncer no Brasil / Instituto Nacional de Câncer José Alencar Gomes da Silva. In 2017. Ministério da saúde, Instituto Nacional de Câncer José Alencar Gomes da Silva (INCA).

29. Chan MH, Wang F, Mang W kong, Tse LA. Sex Differences in Time Trends on Incidence Rates of Oropharyngeal and Oral Cavity Cancers in Hong Kong. Ann Otol Rhinol Laryngol. 2018; 127 (12): 895-902.

30. Momares B, Contreras G, Martínez B, Ávalos N, Carmona L. Sobrevida en Carcinoma Espinocelular de mucosa oral: análisis de $161 \mathrm{pa-}$ cientes. Rev Chil Cir. 2014; 66 (6): 568-76.

31. Shridhar K, Rajaraman P, Koyande S, Parikh PM, Chaturvedi P, Dhillon PK, Rajesh Dikshit P. Trends in mouth cancer incidence in Mumbai, India (1995-2009): An age-period-cohort analysis. Trends mouth cancer Incid Mumbai. 2016; 42 (2016): 66-71.

32. Salgado-Ramíre B, Rivera-Martínez CG, Altamirano-Díaz I, Salgado-Ramírez B. Frecuencia de carcinoma de cavidad oral en un Hospital de tercer nivel. Rev Sanid Milit Mex. 2014; 68 (3): 172-6. 
33. Álvarez Arias D, Munyo Estefan A, Borche G, Rodríguez Sande V, Cuello M. Cáncer de cabeza y cuello en Uruguay. Análisis de sobrevida en dos centros de referencia. Rev Méd Urug. 2018; 34 (1): 21-8.

34. Beovide Cortegoso AV, Koerich Laureano N, Dutra da Silva A, Krebs Danilevicz C, Sellinger Magnusson A, et al. Cell proliferation markers at the invasive tumor front of oral squamous cell carcinoma: comparative analysis in relation to clinicopathological parameters of patients. J Appl Oral Sci. 2017; 25 (3): 318-23.

35. Brandizzi D, Gandolfo M, Velazco ML, Cabrini RL, Lanfranchi HE. Clinical features and evolution of oral cancer: A study of 274 cases in Buenos Aires, Argentina. Med Oral Patol Oral Cir Bucal. 2008; 13 (9): E544-8.

36. Siriwardena BS, Tilakaratne A, Amaratunga EAPD, Tilakaratne WM. Demographic, aetiological and survival differences of oral squamous cell carcinoma in the young and the old in Sri Lanka. Oral Oncol. 2006; 42 (8): 831-6.

37. Muradás Girardi F, Fuga GR, Callai T, Fernandes Bianchi M, Girardi FM. Histopatological differences between genders in patients witH oral squamous cell carcinoma. Clin Biomed Res. 2017; 37 (3): 147-50.

38. Jie W, Bai J, Li B. Clinicopathologic Analysis of Oral Squamous Cell Carcinoma After 125 I Interstitial Brachytherapy. Technol Cancer Res Treatmen. 2018; 17: 1-5.

39. Saira, Fiaz Khan M, Rauf Khattak M, Rawail A, Malik S. Epidemiological and clinical correlates of oral squamous cell carcinoma in patients from north-west Pakistan. J Pak Med Assoc. 2019; 69 (8): 1074-1078.
40. Gul H, Asif F, Ghaffar I, Anwar MA, Tayyab MA, Kashif M. Epidemiology and pathological trends in oral squamous cell carcinoma in a local tertiary care hospital. Int J Community Med Public Heal. 2017; 4 (12): 4440.

41. Leite AA, Leonel ACL da S, de Castro JFL, Carvalho EJ de A, Vargas PA, Kowalski LP, da Cruz Perez DE. Oral squamous cell carcinoma: A clinicopathological study on 194 cases in northeastern Brazil. A cross-sectional retrospective study. Sao Paulo Med J. 2018; 136 (2): 165-9.

42. Álvarez Martínez E, Preciado A, Montoya Fernández SA, Jiménez Gómez R, Posada A. Características clínico-histopatológicas del carcinoma escamocelular bucal, Colombia. Rev Cubana Estomatol. 2010; 47 (1): 81-95.

43. Bórquez PM, Capdeville FF, Madrid AM, Veloso MO, Cárcamo MP. Sobrevida global y por estadíos de 137 pacientes con cáncer intraoral. Experiencia del Instituto Nacional del Cáncer. Rev. Chilena de Cirugía. 2011; 63 (4): 351355.

44. Bonfante GM, Machado CJ, Souza PEA, Andrade EIG, Acurcio F de A, Cherchiglia ML. Sobrevida de cinco anos e fatores associados ao câncer de boca para pacientes em tratamento oncológico ambulatorial pelo Sistema Único de Saúde, Brasil. Cad Saude Publica. 2014; 30 (5): 983-97.

45. Brandizzi D, Gandolfo M, Velazco ML CR, Lanfranchi HE. Clinical features and evolution of oral cancer: a study of 274 cases in Buenos Aires, Argentina. Med Oral Patol Oral Cir Bucal. 2008; 13: E544-8.

Natalia González Umpiérrez: nataliagonzalez@odon.edu.uy 